\title{
A New Dynamical Diffraction-Based Technique of the Residual Stress Measurements in Thin Films
}

\author{
M. Agamalian, ${ }^{1}$ E. Iolin, ${ }^{1}$ H. Kaiser, ${ }^{2}$ Ch. Rehm, ${ }^{1}$ and S. A. Werner ${ }^{2,3}$ \\ ${ }^{1}$ Oak Ridge National Lab. Current location: Argonne National Lab, Argonne, IL 60439 \\ ${ }^{2}$ University of Missouri-Columbia, Columbia, MO 65211 \\ ${ }^{3}$ National Institute of Standards and Technology, Gaithersburg, MD 20899
}

\begin{abstract}
The recently discovered dynamical diffraction effect "neutron camel" was used for the residual stress measurements in a thick $\operatorname{Si}(111)$ crystal coated with a $2000 \AA$ thick Ni film. The observed asymmetry of the back-face rocking curve corresponds to the bending radius of $\sim 19 \mathrm{~km}$ and the tension force applied to the $\mathrm{Ni}$ film is $\sim 90 \mathrm{~N} / \mathrm{m}$. Relative deformation of the Si crystallographic cells in the vicinity of diffractive surfaces is $\left|\partial \mathrm{u}_{\mathrm{z}} / \partial \mathrm{z}\right| \approx 1.6 \cdot 10^{-6}$.
\end{abstract}

PACS numbers: 62.20.-x, 61.12.Ex, 68.60.-p

\section{INTRODUCTION}

In the past two decades optical devices, consisting of thin reflecting layers deposited on silicon or silicon dioxide substrates, have found wide application in light, Xray and neutron diffraction. A significant surface-induced residual stress, which usually remains in the films as well as in the substrates after deposition, creates a serious limitation of quality of these devises. The residual stress in crystalline films can be 
detected directly by the conventional X-ray diffraction technique [1]. There exists another laser-based in situ technique, Surface-Stress-Induced Optical Deflection (SSIOD), which is able to detect small deformation strain in substrates during the coating process [2]. The Back-Face Neutron Diffraction (BFND) from a perfect Si crystal, as it was shown in [3, 4], is extremely sensitive to the ultra-small deformation strain. This technique is capable of detecting residual stress in single crystals even when the relative deformation of the crystallographic cells is as small as $\sim 8 \cdot 10^{-7}$, which corresponds to the radius of bending of $\sim 40 \mathrm{~km}$. The radius of bending can be converted to the value of deformation of crystallographic cells in the film in a manner similar to the SSIOD technique [2], however in contrary BFND works for final products. In the present work we describe the first successful attempt to apply the BFND technique for detecting ultra-small residual stress in a thick Si crystal coated with a thin Ni film.

\section{INSTRUMENTS AND SAMPLES}

The experiments were carried out using the Double-Crystal Diffractometer at Oak Ridge National Laboratory [5] in the geometry similar to that described in [3] (see Fig. 1). The primary beam was diffracted from a Si(111) mosaic [22' FWHM] crystal, then from a $\mathrm{Si}(111)$ pre-monochromator (both are not shown in Fig. 1), and finally from a $\operatorname{Si}(111)$ triple-bounce channel-cut monochromator. The wavelength was $\lambda=2.59 \AA$; the beam cross-sectional area was $2 \mathrm{~cm} \times 4 \mathrm{~cm}$. The primary beam was restricted with a fixed vertical $1.8 \mathrm{~mm}$ wide $\mathrm{Cd}$ slit and the second scanning $4 \mathrm{~mm}$ wide slit was mounted directly in front of the detector. In this configuration, the intensity diffracted from different volumes within the crystal was mapped and the transmitted beam was used as a 
monitor signal for determination of the exact Bragg angle, $\theta_{\mathrm{B}}$, when the rocking curves were measured for positions other than the front face of the crystal.

A perfect $\mathrm{Si}(111)$ slab-shaped crystal with dimensions $114 \mathrm{~mm}$ x $40 \mathrm{~mm}$ x 8.19 $\mathrm{mm}$ was cut with the $114 \mathrm{~mm} \times 40 \mathrm{~mm}$ diffractive surfaces parallel to the (111) crystallographic planes for evaluation of the diffraction in the near vicinity of Bragg reflection. The diffractive surfaces were polished mechanically, etched, and finally polished chemically. The surface metrology measurements were carried out using a WYKO laser figure interferometer. The root mean square height variation is $1.74 \mu \mathrm{m}$ with the maximum peak to valley value of $7.88 \mu \mathrm{m}$. The Front Face (FF), Back Face (BF) and Garland Rocking Curves (FFRC, BFRC and GRC) shown in Fig. 1 were measured after the surface treatment. Then, one of the diffractive surfaces of the crystal was coated with a $2000 \AA$ thick Ni film using a magnetron sputtering technique, and the neutron diffraction measurements were undertaken again.

\section{THEORETICAL BACKGROUND}

It is known that in the near vicinity of the exact Bragg angle neutron waves propagate inside the thick perfect crystal in the direction parallel to the diffractive surfaces by sequential reflection from the front and back faces (BF-FF-BF- mode). When the crystal is lightly deformed, the neutron trajectories inside the crystal become curved, which leads to appearance of the second, additional to the BF-FF-BF-, mode of propagation created by so-called garland reflections [7] only from the FF (see Fig. 2). The primary monochromatic beam defined with a narrow cadmium slit partly reflects from the FF of the crystal at $\mathrm{X}=0$ and the other part penetrates inside and splits on the BF-FF-BF- mode (see the trajectories $0-4-5$ and $0-6-7$ ), and by the garland mode (the 
trajectories $0-1,0-2$, and $0-3$ ). The curved trajectories $0-4-5$ and $0-6-7$ become strait when the crystal is not deformed, thus the garland reflections are eliminated. Fig. 3 clearly shows that the first back-face reflection measured in our experiment (see the position 7 in Fig. 1) mostly contains the admixture of one-bounce garland reflection (see the peak 1 in Fig. 2), which gives significant contribution to the corresponding BFRC. The $B F, R_{B F}(y)$, and garland, $\mathrm{R}_{\mathrm{G}}(\mathrm{y})$, reflectivity functions were derived from the Takagi-Taupin equations $[8,9]$, which in the case of cylindrical deformation can be significantly simplified [3]. Here $\mathrm{y}=\left(\theta-\theta_{\mathrm{B}}\right) / \delta \theta_{\mathrm{D}}$ is the dimensionless angular parameter of the dynamical diffraction theory and $\delta \theta_{\mathrm{D}}$ is a half-width of the Darwin plateau [6]. Our model contains only one independent parameter of deformation $b \sim \partial^{2}(\mathbf{H U}) / \partial \mathrm{s}_{0} \partial \mathrm{s}_{\mathrm{h}}$, which is proportional to the gradient of the lattice constant. Here $\mathbf{H}$ is the vector of scattering, $H=4 \pi \sin \theta_{\mathrm{B}} / \lambda, \mathbf{U}$ is the displacement of nuclei under the deformation force, and $\mathrm{s}_{0}=\left(\mathrm{X} / \cos \theta_{\mathrm{B}}+\mathrm{Z} / \sin \theta_{\mathrm{B}}\right) / 2$, $\mathrm{S}_{\mathrm{h}}=\left(\mathrm{X} / \cos \theta_{\mathrm{B}}-\mathrm{Z} / \sin \theta_{\mathrm{B}}\right) / 2$ are the coordinates directed along the incident and diffracted beams correspondingly. $R_{G}(y)$ was calculated numerically and $R_{B F}(y)$, for $b>0, y<-1$, and $\mathrm{b}>0, \mathrm{y}>1+2 \mathrm{bT}$ (where $\mathrm{T}=\left(\mathrm{T}_{1} / \tau\right) \cdot \pi \cdot \operatorname{ctg} \theta_{\mathrm{B}}$ is the dimensionless crystal thickness, $\mathrm{T}_{1}$ is the crystal thickness in $\mu \mathrm{m}$, and $\tau \approx 77 \mu \mathrm{m}$ is the extinction length) obtained in analytical form using the geometrical optics approximation. Fig. 3 shows the total reflectivity functions, $\mathrm{R}(\mathrm{y})=\mathrm{R}_{\mathrm{BF}}(\mathrm{y})+\mathrm{R}_{\mathrm{G}}(\mathrm{y})$, integrated over the detector slit $\mathrm{X}_{1}, \mathrm{X}_{2}$ (Fig. 2). The discrete spectrum of $\mathrm{R}_{\mathrm{G}}(\mathrm{y})$ (see peaks 1, 2 and 3 in Fig. 3) appears only due to the chosen geometry of the experiment. The experimentally measurable BFRC (see positions 7 in Fig. 1) is the convolution of the Darwin reflectivity function of the triple-bounce monochromator, $\mathrm{R}_{\mathrm{D}}^{3}(\mathrm{y})$, with the total reflectivity, $\mathrm{R}(\mathrm{y})$ :

$$
\mathrm{I}(\Delta)=\int \mathrm{R}_{\mathrm{D}}^{3}(\mathrm{y}) \cdot \mathrm{R}(\mathrm{y}+\Delta) \mathrm{dy},
$$


If the crystal is not deformed, $\mathrm{R}_{\mathrm{G}}(\mathrm{y})=0$ and $\mathrm{R}(\mathrm{y})=\mathrm{R}_{\mathrm{BF}}(\mathrm{y})$. If the crystal is lightly deformed, $\mathrm{R}(\mathrm{y})=\mathrm{R}_{\mathrm{G}}(\mathrm{y})$ for the position 6 (Fig. 1) and $\mathrm{R}(\mathrm{y})=\mathrm{R}_{\mathrm{G}}(\mathrm{y})+\mathrm{R}_{\mathrm{BF}}(\mathrm{y})$ for the position 7 .

\section{RESULTS AND DISCUSSION}

The experimental rocking curves as well as the appropriate theoretical simulations are given in Fig. 4. The experimental BFRC obtained from the Si crystal without the Ni film (is not shown) is symmetric with respect to the exact Bragg angle, $\theta-\theta_{\mathrm{B}}=0$, however the dramatic change in the BFRC has been observed for the same crystal after depositing the $2000 \AA$ Ni film on one of the diffractive surfaces (see Fig. 4). The BFRCs 1 and 2 (Fig. 4), measured for two orientations of the crystal (when the Ni coated surface is set up as the BF (1) and then as the FF (2)), are strongly asymmetrical, and in the cylindrical deformation approximation can be considered as the mirror reflections of each other. It is worthwhile to note here that the two diffractive surfaces are not in principle optically identical due to presence of the Ni film on one of them. However, the Bragg angle, $\theta_{\mathrm{B}}=24.4^{\circ}$, is much larger than the critical angle of total reflection from $\mathrm{Ni} /$ air interface $\left(\theta_{\mathrm{c}}=0.25^{\circ}\right.$ for $\lambda=2.59 \AA$ ), which completely excludes any possibility of mirror reflection from the Ni film. Also, the absorption and any type of scattering from $2000 \AA$ Ni film are vanishing small compared to the intensity of BF diffraction. The theoretical BFRCs, calculated by formulas (5) for the parameters $b \approx 4 \cdot 10^{-4}$ and $b \approx 3.5 \cdot 10^{-4}$, fit quite well to the corresponding experimental rocking curves. However, the experimental BFRCs do not contain the tiny peaks and steps resulting from the convolution of the main central peak of the BF reflectivity function (see Fig. 3) with that for the triple-bounce monochromator. This difference can easily be explained by the fact that the minimal 
angular step of the ORNL double-crystal diffractometer, $\Delta\left(\theta-\theta_{\mathrm{B}}\right)=0.25$ arcsec, is too large to resolve these tiny peculiarities of the BFRC profile. However, the central peak, which corresponds to the strongest one-bounce garland reflection, contributes significantly to the intensity of the rocking curve and increases its asymmetry by $\sim 35 \%$. The parameter $\mathrm{b} \approx 4 \cdot 10^{-4}$, determined from the best fits of the experimental BFRC, corresponds to the relative deformation of the Si crystallographic cells in the vicinity of diffractive surfaces, $\left|\partial \mathrm{u}_{\mathrm{z}} / \partial \mathrm{z}\right| \approx 1.6 \cdot 10^{-6}$, and a radius of bending, $\mathrm{R}_{\mathrm{b}} \approx(\mathrm{H} / 2 \mathrm{~b})(\tau / \pi)^{2} \approx 19$ $\mathrm{km}$, where $\tau$ is the extinction length [3]. The Stoney formula [1,2] converts the value of $\mathrm{R}_{\mathrm{b}}$ to the tension force, $f$, applied to the film as a result of the substrate deformation:

$$
f=\mathrm{ET}^{2} /\left[6\left(1-v^{2}\right) \mathrm{R}_{\mathrm{b}}\right]
$$

where $\mathrm{E} \sim 10^{12} \mathrm{dyn} / \mathrm{cm}^{2}$ is the modulus of elasticity, and $v$ is the Poisson constant of Si. Thus, the calculated value of $f$ is $\sim 90 \mathrm{~N} / \mathrm{m}$, which corresponds to deformation of the $\mathrm{Ni}$ crystallographic cells $\left|\partial \mathrm{u}_{\mathrm{x}} / \partial \mathrm{x}\right| \approx 2 \cdot 10^{-3}$. Thus, the $2000 \AA \mathrm{Ni}$ film under study is strongly strained (expanded along the $\mathrm{X}$ axis, Fig. 2). The described experiments clearly show that the BFND, which is extremely sensitive to the ultra-small deformation strain in the crystal, can be used for residual stress measurements in thin films deposited on the diffractive surface. The BFND works in principle similarly to the SSIOD in situ technique, detecting the deformation of the substrate, thus, it is capable to measure residual stress not only in crystalline, likewise the X-ray diffraction technique, but also in any amorphous, polymer, colloidal, mono- and multilayer thin films deposited on the diffractive surface of Si single crystals. The BFND, however, is not an in situ technique 
and it allows evaluating the final product. We, therefore, expect its broad application, particularly in characterization of neutron and X-ray optical devices and in the semiconductor industry.

The authors thank L. Assoufid and R Khachatryan (Argonne National Lab) for surface metrology measurements. The submitted manuscript has been authored by a contractor of the U.S. Government under contract No. DE-AC05-960R22464. Accordingly, the U.S. Government retains a nonexclusive, royalty-free license to publish or reproduce the published form of this contribution, or allow others to do so, for U.S. Government purposes. This research was in part supported by the Postdoctoral Research Associates Program administrated jointly by Oak Ridge National Laboratory and Oak Ridge Institute for Science and Education and the National Science Foundation. E. Iolin acknowledges support of a COBASE grant of the National Research Council of Missouri. S. A. Werner's work on the project is supported by NSF-PHY-9603559.

[1] I. C. Noyan, T. C. Huang, and B. R. York: Critical Reviews in Solid State and Materials Sciences 20(2), 125 (1995)

[2] M. Bicker, U. von Hülsen, U. Laudahn, A. Pundt, and U. Geyer: Rev. Scientific Instr. 69, 460 (1998)

[3] M. Agamalian, E. Iolin, L. Rusevich, C. J. Glinka, and G. D. Wignall: Phys. Rev. Lett. 81, 602 (1998)

[4] M. Agamalian, E. Iolin and G.D. Wignall: Neutron News 10, 24 (1999)

[5] M. Agamalian, R. Triolo, and G. D. Wignall: J. Appl. Crystallogr. 30, 345 (1997) 
[6] W. H. Zachariasen: Theory of X-rayDiffraction in Crystals (Dover Publ.,NY,1967)

[7] F. N. Chukhovskii and P. V. Petrashen: Acta Crystallogr A. 44, 8 (1988)

[8] S. Takagi: Acta Crystallogr. 15, 1311 (1962)

[9] D. Taupin: Bull. Soc. Fr. Mineral. Crystallogr. 84, 51 (1961)

\section{FIGURE CAPTIONS}

1. Geometry of the experiment: 1 is the triple-bounce monochromator; 2, 4 are immovable and scanning $\mathrm{Cd}$ slits correspondingly; 3 is the $\operatorname{Si}(111)$ slab-shaped crystal coated with a $2000 \AA$ Ni film; 5, 6, 7 are the FF, Garland and BF reflections correspondingly; and 8 is the transmitted beam used as a monitor signal.

2. The BF, 0-4-5, 0-6-7, and the garland, 0-1,0-2,0-3, trajectories in the deformed crystal; $\mathrm{X}_{1}, \mathrm{X}_{2}$ are the coordinates of the detector window aligned with respect to the $\mathrm{BF}$ reflected beam; and $\mathrm{T}$ is the crystal thickness.

3. The theoretical BF reflectivity curve numerically calculated for the deformed crystal with $\mathrm{T}=8.19 \mathrm{~mm}$ and $\mathrm{b} \approx 4 \cdot 10^{-4} \cdot 1,2$, and 3 are the diffraction peaks generated by the single-, double- and triple-bounce garland trajectories correspondingly; 4 are the peaks induced by the BF, 0-4-5, 0-6-7, trajectories in Fig. 2.

4. The BFRCs measured after coating with the $2000 \AA \mathrm{Ni}$ film: 1 - the Ni film is on the BF, as shown in Fig. 1;2 - the Ni coated surface is on the FF. Solid and dashed lines are the simulation curves calculated for $b=4 \cdot 10^{-4}$ and $b=3.5 \cdot 10^{-4}$ correspondingly. 


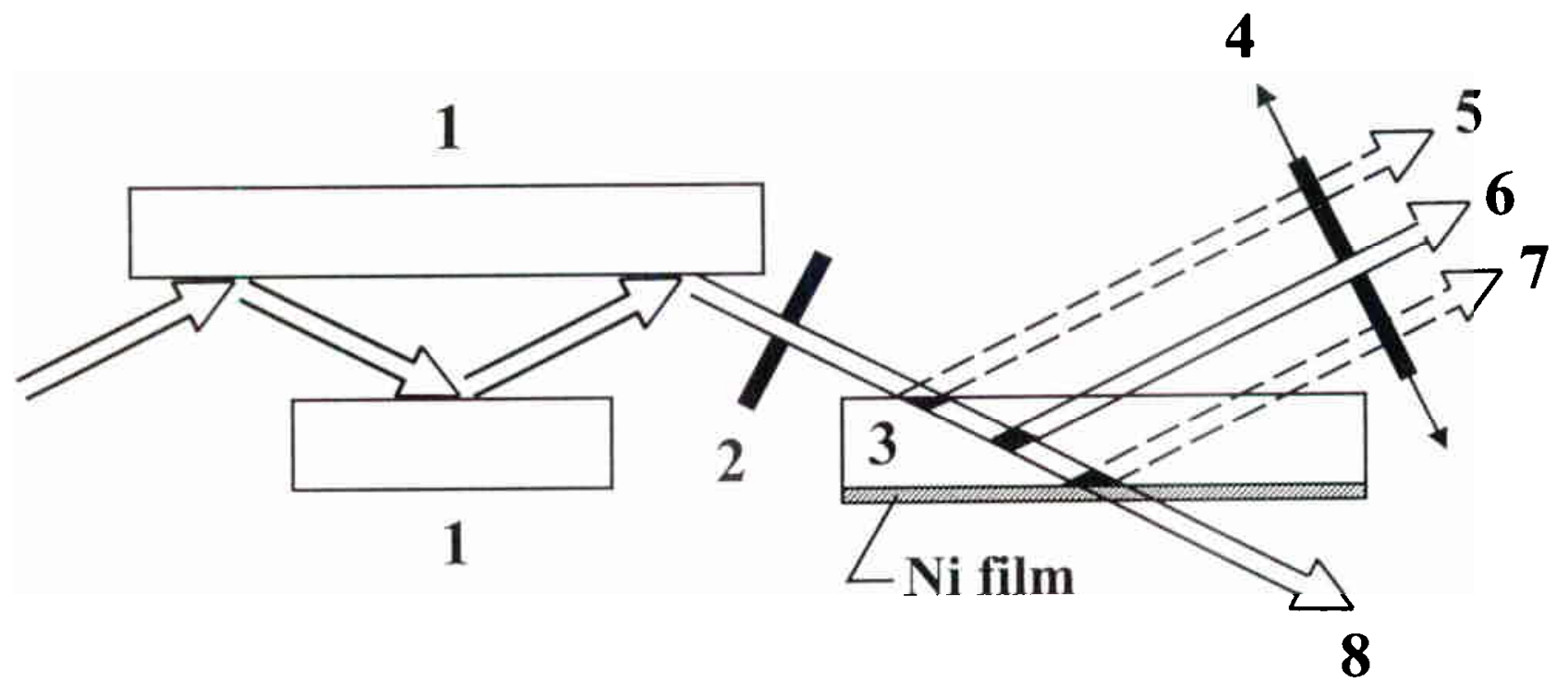

Fig. 1 


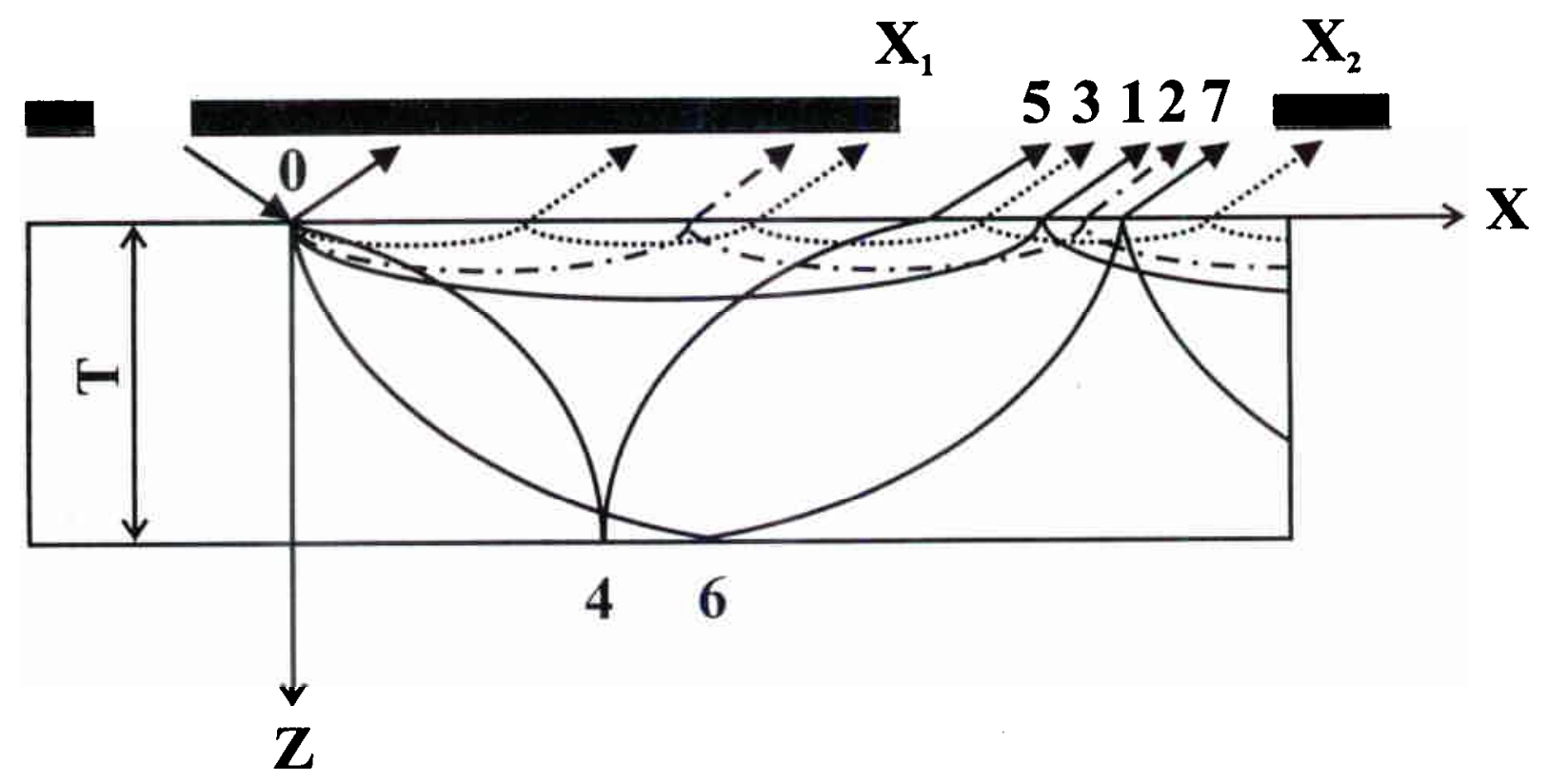

Fig. 2 


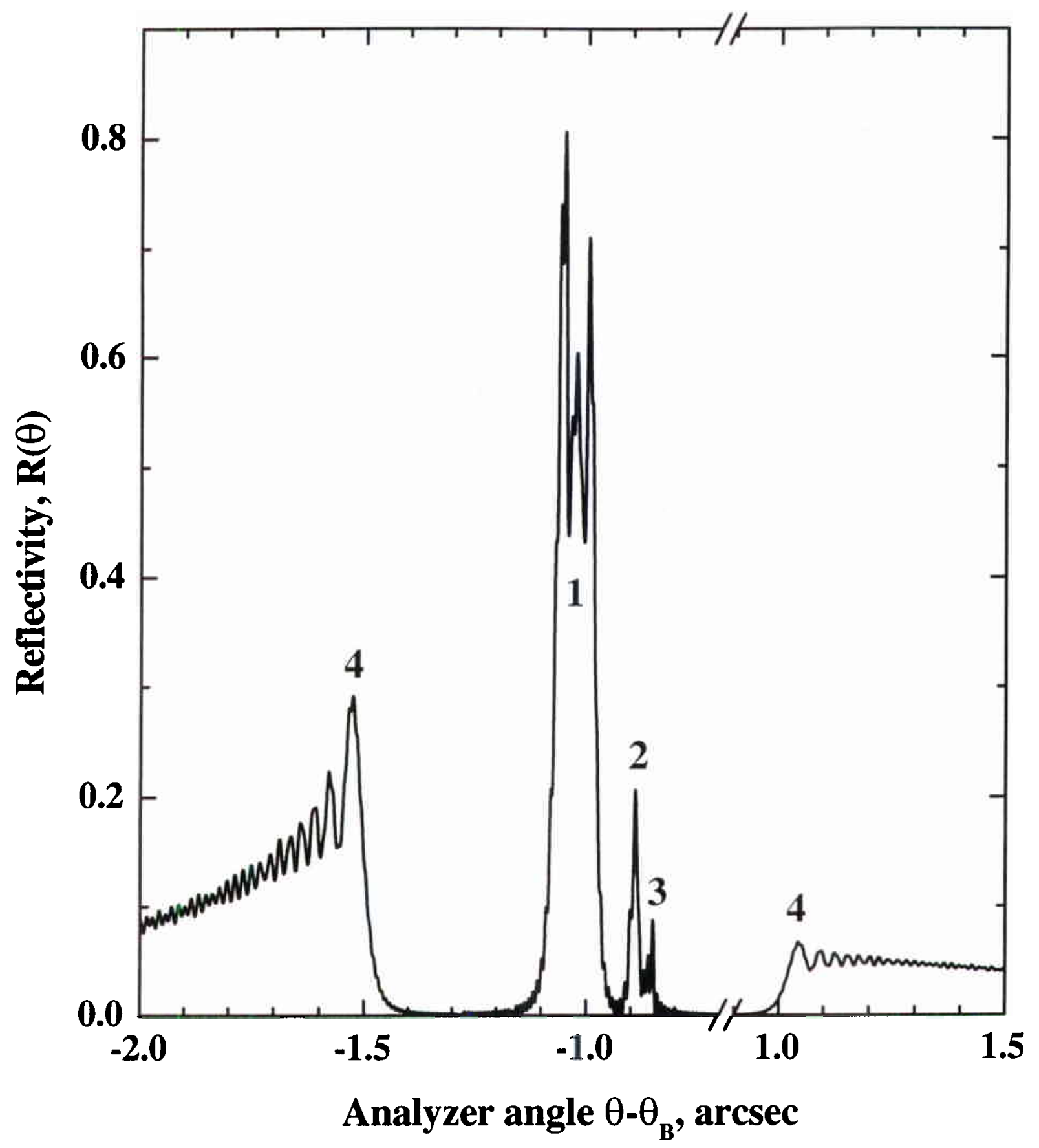

Fig. 3 


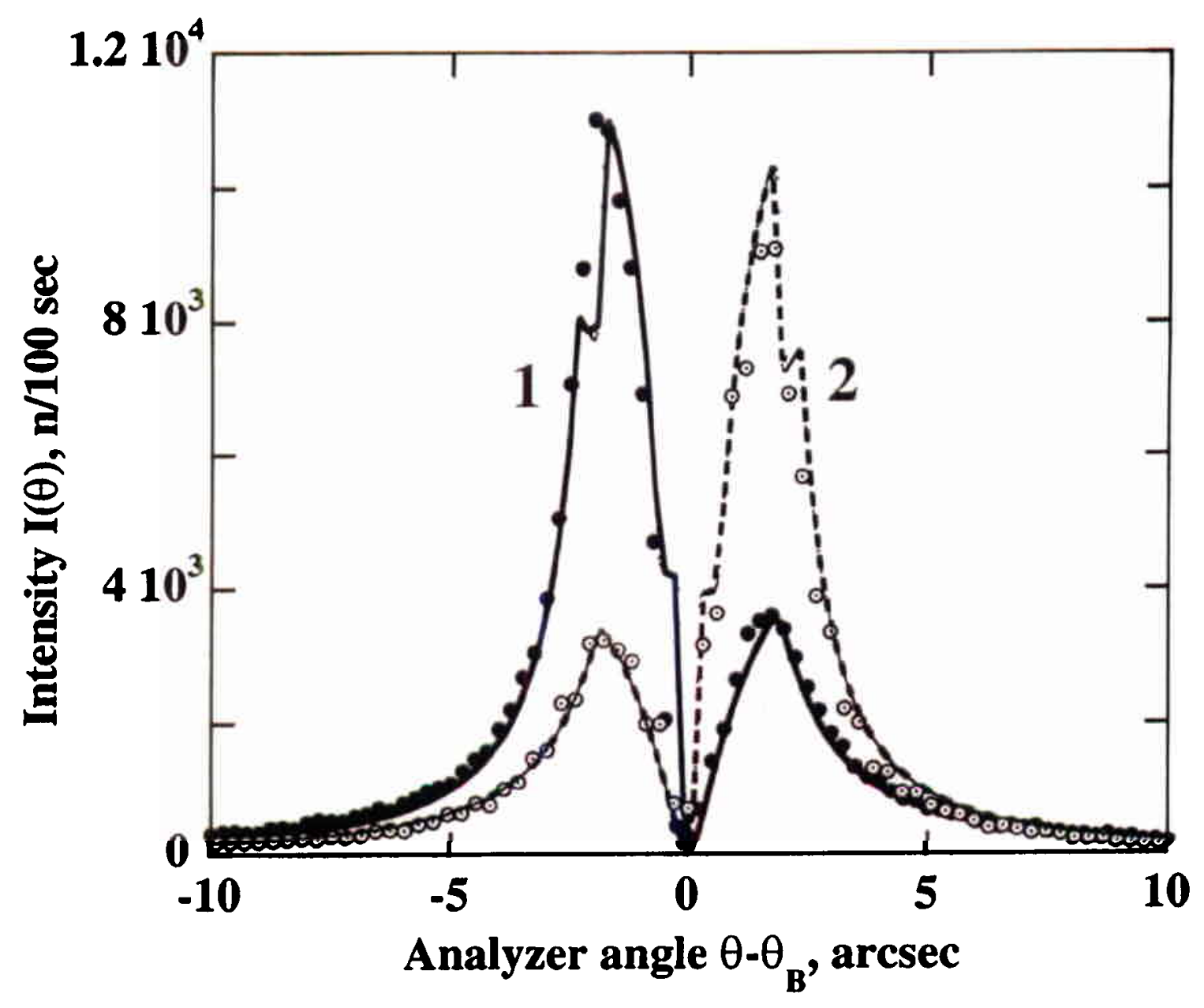

Fig. 4 